\title{
FLT3 Internal Tandem Duplication Positive
}

National Cancer Institute

\section{Source}

National Cancer Institute. FLT3 Internal Tandem Duplication Positive. NCI Thesaurus.

Code C150626.

An indication that internal tandem duplication within the FLT3 gene has been detected in a sample. 